\title{
Optimality of the dynamic procedure for large-eddy simulations
}

\author{
Johan Meyers ${ }^{\text {a) }}$ \\ Department of Mechanical Engineering, Katholieke Universiteit Leuven, Celestijnenlaan 300, \\ B3001 Leuven, Belgium \\ Bernard J. Geurts ${ }^{\text {b) }}$ \\ Mathematics of Turbulence and Complex Fluids, Faculty EEMCS, University of Twente, P.O. Box 217, \\ 7500 AE Enschede, The Netherlands and Department of Applied Physics, Fluid Dynamics Laboratory, \\ Technical University of Eindhoven, P.O. Box 513, 5600 MB Eindhoven, The Netherlands \\ Martine Baelmans ${ }^{\mathrm{c})}$ \\ Department of Mechanical Engineering, Katholieke Universiteit Leuven, Celestijnenlaan 300, \\ B3001 Leuven, Belgium
}

(Received 16 August 2004; accepted 27 January 2005; published online 31 March 2005)

\begin{abstract}
We present a database analysis to obtain a precise evaluation of the accuracy limitations associated with the popular dynamic eddy-viscosity model in large-eddy simulation. We consider decaying homogeneous isotropic turbulence at two different Reynolds numbers, i.e., $\operatorname{Re}_{\lambda}=50$ and 100 . The large-eddy simulation errors associated with the dynamic model are compared with those arising in the "static" Smagorinsky model. A large number of systematically varied simulations using the Smagorinsky model provides a detailed impression of the dependence of the total simulation error on (i) the spatial resolution and (ii) the resolution of the subgrid dissipation length. This error behavior also induces an "optimal refinement trajectory" which specifies the particular Smagorinsky parameter, in terms of the spatial resolution, for which the total error is minimal. In contrast, the dynamic model gives rise to a self-consistently determined "dynamic trajectory" that represents the dependence of the dynamic coefficient on the spatial resolution. This dynamic trajectory is compared with the optimal refinement trajectory as obtained from the full database analysis of the Smagorinsky fluid. It is shown that the dynamic procedure in which the top-hat test filter is adopted, predicts values for the eddy viscosity as function of resolution and Reynolds number, which quite closely follow the main trends established in the optimal refinement trajectory. Furthermore, a sensitivity analysis, including dependency on test-filter width and filter shape, is discussed. Total simulation errors, due to interacting discretization, and modeling errors associated with the dynamic procedure may be a factor 2 higher compared to the optimum; still the dynamic procedure represents one of the very few self-contained and efficient error-reduction strategies when increasing the spatial resolution. (C) 2005 American Institute of Physics. [DOI: 10.1063/1.1879054]
\end{abstract}

\section{INTRODUCTION}

Associated with the continuing development of modern computer technologies, computational fluid dynamics can play a steadily increasing role of importance in fundamental fluid mechanics research as well as in industrial design and control processes. In particular, large-eddy simulation (LES) is evolving into a major simulation technique for turbulent flows, with the potential to combine cost effectiveness with accuracy. ${ }^{1,2}$ In LES, only the dominant, large-scale features of turbulent flow fields are directly computed using the spatially filtered Navier-Stokes equations. The small-scale features that are characteristic for a turbulent flow are explicitly removed from the description by the low-pass filter that is applied. However, the dynamic influence of these small-scale features on the evolution of the resolved large-scale aspects of the flow is explicitly represented by the introduction of a so-called subgrid scale model. This model expresses a pa-

\footnotetext{
${ }^{a}$ Electronic mail: Johan.Meyers@mech.kuleuven.ac.be

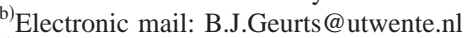

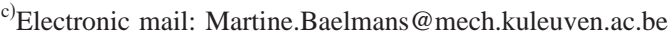

rametrization for the central closure problem that arises from filtering the nonlinear terms in the Navier-Stokes equations. Specifically, the closure problem is expressed in terms of the turbulent stress tensor.

In "LES history," many different subgrid scale models have been proposed and tested. A detailed overview is, e.g., provided in Ref. 1. Both mathematical as well as physical arguments have been put forward in order to justify specific subgrid models. These emphasize, in particular, the capturing of the basic dispersive and dissipative contributions from the subgrid scale dynamics. ${ }^{3}$ Certainly, the most commonly used model in LES practice and often adopted as point of reference for constructing or comparing new models is the Smagorinsky model. ${ }^{4}$ This eddy-viscosity model contains a model coefficient-the Smagorinsky constant $C_{S}$-which needs to be provided by the user, i.e., externally specified. In principle, a "universal" value for the Smagorinsky constant can be suggested provided additional assumptions about the nature of the turbulent flow are invoked. ${ }^{5,6}$ However, simulations using these "theoretical" values for $C_{S}$ are often found to be rather inaccurate, e.g., because the model is 
found to be too dissipative. ${ }^{7}$ Therefore, in practice, the Smagorinsky constant is often treated as an ad hoc parameter in the model, which is adapted according to specific application related requirements. Obviously, this does not contribute to the predictive capabilities of this model and various extensions are known in literature.

A strategy for removing most of the external "arbitrariness" related to ad hoc coefficients in specific subgrid scale models is provided by the well-known dynamic procedure. ${ }^{8-10}$ This procedure allows to determine model coefficients in accordance with properties of the evolving resolved flow solution. The dynamic procedure yields space and time dependent coefficients which are such that the correspondence of the assumed model with the Germano identity ${ }^{9}$ for the turbulent stress tensor is optimal. The dynamic procedure has been widely applied to a variety of base models, among others, Refs. 11-13. Historically, the dynamic procedure was first adopted in combination with the Smagorinsky base model and shown not only to remove the existing arbitrariness in the base model but also to considerably improve the accuracy of the predictions. Although some ad hoc implementation aspects, such as (local) averaging and "clipping" 14 of the "raw" model coefficients remain in the dynamic Smagorinsky model, generally, this model has become very popular as it often combines acceptable accuracy with significant robustness of the simulation, even at very high Reynolds numbers and on comparably coarse grids used in computations. ${ }^{15}$

The accuracy of actual LES predictions in general not only depends on the quality of the subgrid model, but also on a number of additional factors that are part of the computational modeling of a flow. In fact, further complications may arise from the specific flow geometry, from the flow regime, from the mesh and mesh size, and from the numerical method. Moreover, all these contributions are likely to lead to intricate dynamical interactions which may induce a nonlinear accumulation of errors in a simulation. Ideally speaking, a hypothetical "optimal" dynamical procedure should account for these different influences and automatically adapt model coefficients in order to arrive at the smallest total error for a prespecified computational cost. To what extent actual implementations of the "traditional" dynamic procedure already provide such efficient error reduction with increased computational cost, e.g., when allowing the spatial resolution to increase, is the main subject of this paper. We will concentrate on a recently developed database approach to identify the global error behavior as function of, e.g., spatial resolution, model parameters, and flow conditions. ${ }^{16}$

Central to a framework for assessing the error behavior associated with an actual implementation of the dynamic procedure is the evaluation of the total simulation error and its decomposition into numerical and modeling components. For the definition of the total simulation error, the reference is provided by results obtained from direct numerical simulation (DNS) of the same flow and under identical flow conditions. By appropriately comparing LES with DNS predictions for a number of flow properties, e.g., the kinetic energy evolution or its spectrum, the total simulation errors can be quantified. ${ }^{16,17}$
For the specific purpose of assessing the error behavior of the dynamic procedure the central focus is on the so-called "accuracy-chart" and the reference "optimal refinement strategy" introduced in Ref. 16. In particular, LES of homogeneous, decaying turbulence was studied in which the basic Smagorinsky subgrid model ${ }^{4}$ was employed. We recall that the accuracy chart for such a "Smagorinsky fluid" is a representation of the total simulation error at some flow conditions, as function of spatial resolution $N$ and the resolution of the Smagorinsky length, $\xi_{S}=\ell_{S} / h$, where $h=1 / N$ is the mesh spacing and $\ell_{S}$ is the "Smagorinsky length" given by $\ell_{S}$ $=C_{S} \bar{\Delta}$, with $C_{S}$ the Smagorinsky constant and $\bar{\Delta}$ the LES filter width. Each point in the $\xi_{S}-N$ plane corresponds to a particular large-eddy simulation of the Smagorinsky fluid which displays its own specific deviation from the exact DNS results. An "error landscape" is created by considering the simulation error for a systematically varied set of $\xi_{S}-N$ points, leading to an extensive database approach to assessing the error behavior. In this accuracy chart the line $\hat{\xi}_{S}(N)$ for which the simulation error is minimal represents the optimal refinement strategy for the Smagorinsky model. By adapting $\xi_{S}$ (i.e., $C_{S} \bar{\Delta} / h$ ) in this optimal way to changes in the spatial resolution one may a posteriori take all interacting modeling and discretization error effects into consideration.

The error dynamics associated with the dynamic procedure may readily be interpreted in relation to this accuracy chart; the dynamic procedure gives a priori rise to a selfinduced dynamic coefficient $C_{d}(N)$, which is readily translated into a "dynamic refinement trajectory" $\xi_{d}(N)$ $=\bar{\Delta} \sqrt{C_{d}(N)} / h$ on the $\xi_{S}-N$ plane, along which a total simulation error as function of spatial resolution $N$ is induced. Of particular importance is, of course, how this "dynamic trajectory" $\xi_{d}(N)$ relates to the optimal refinement strategy $\hat{\xi}_{S}(N)$, i.e., the ability of the dynamic procedure to approximate the optimal error line. In this paper we will address this aspect of the dynamic procedure in detail and establish the efficiency of the total error reduction induced by this model when the grid is refined.

The organization of this paper is as follows. In Sec. II, the large-eddy equations are presented. Moreover, we detail the implementation of the dynamic procedure. Subsequently, Sec. III compares "dynamic trajectories" with "optimal refinement trajectories." A sensitivity analysis, which incorporates variations due to changes in the width or in the shape of the test filter are discussed in Sec. IV. Finally, some concluding remarks are presented in Sec. V.

\section{GOVERNING EQUATIONS}

In this section we present the unclosed LES equations, identify the central closure problem and specify the basic Smagorinsky subgrid model that was adopted to represent the turbulent stress tensor. Moreover, we describe the dynamic procedure ${ }^{8}$ used in combination with the Smagorinsky model and introduce the error-assessment approach in terms of "coefficient trajectories" on an "accuracy chart," which will be defined in some detail. 
In order to arrive at the spatially filtered representation of a turbulent flow, we introduce the filter operator $L$. On an unbounded domain in one spatial dimension we write

$$
\bar{u}(x, t)=L(u)(x, t)=\int_{-\infty}^{\infty} G(x-\xi) u(\xi, t) d \xi
$$

where $\bar{u}$ denotes the filtered velocity, $u$ the unfiltered velocity, and $G$ the normalized filter kernel, i.e., $L(c)=c$ for any constant function $c$. In three dimensions the filter operator can be constructed as the composition of three onedimensional filters. The operator $L$ is linear and commutates with partial derivatives. For incompressible flow we hence observe that the filtered solution is also solenoidal, that is, $\partial \bar{u}_{j} / \partial x_{j}=0$ where $\bar{u}_{j}$ denotes the $j$ th velocity component corresponding to the Cartesian coordinate $x_{j}$. Throughout, the summation convention over repeated indices is adopted. The filtered, incompressible Navier-Stokes equations can be written as

$$
\frac{\partial \bar{u}_{i}}{\partial t}+\frac{\partial\left(\bar{u}_{i} \bar{u}_{j}\right)}{\partial x_{j}}+\frac{\partial \bar{p}}{\partial x_{i}}-\frac{2}{\operatorname{Re}} \frac{\partial \bar{S}_{i j}}{\partial x_{j}}=\frac{\partial \tau_{i j}}{\partial x_{j}}, \quad i=1,2,3
$$

in terms of the filtered solution given by $\left\{\bar{u}_{i}, \bar{p}\right\}$, where $\bar{p}$ denotes the filtered pressure. The filtering of the convective terms gives rise to the divergence of the turbulent stress tensor,

$$
\tau_{i j}=\bar{u}_{i} \bar{u}_{j}-\overline{u_{i} u_{j}},
$$

which expresses the central closure problem in LES. In order to arrive at a self-contained system of equations, a particular subgrid model needs to be introduced, approximating the dynamic effects associated with $\tau_{i j}$. Finally,

$$
\bar{S}_{i j}=\frac{1}{2}\left(\frac{\partial \bar{u}_{i}}{\partial x_{j}}+\frac{\partial \bar{u}_{j}}{\partial x_{i}}\right)
$$

denotes the filtered rate of strain tensor.

As basic model we adopt the classical Smagorinsky ${ }^{4}$ subgrid model $m_{i j}^{S}$ to approximately represent $\tau_{i j}$. In particular, this implies

$$
\tau_{i j} \rightarrow m_{i j}^{S}=2\left(C_{S} \bar{\Delta}\right)^{2}|\bar{S}| \bar{S}_{i j}=2 \ell_{S}^{2}|\bar{S}| \bar{S}_{i j},
$$

where $|\bar{S}|=\left(2 \bar{S}_{i j} \bar{S}_{i j}\right)^{1 / 2}$ is the magnitude of the filtered rate of strain tensor and we introduced the Smagorinsky length $\ell_{S}$ $=C_{S} \bar{\Delta}$ for later convenience. The amount by which this eddyviscosity model smoothes the solution, compared to the DNS result, is determined in part by $\ell_{S}$. While it is customary to choose the LES filter width $\bar{\Delta}=h$, possible values for the Smagorinsky constant can be estimated under some additional assumptions concerning the underlying nature of the flow and the shape of the filter. Various values have been suggested, mainly ranging from $C_{S}=0.1$ to $C_{S}=0.2$, e.g., Refs. 5, 18, and 19.

Rather than adhering to a specific value for $C_{S}$, in this paper we will follow an alternative route in which the eddyviscosity coefficient is determined self-consistently during the large-eddy simulation. This is known as the dynamic procedure $^{8-10}$ which is based on general algebraic relations that exist between different filtered representations of a tur- bulent field. Specifically, consider two filters, one with kernel $G$ and filter width $\bar{\Delta}$, and a second (test) filter with kernel $H$ and a filter width $\hat{\Delta}$. Associated with these two filters, two different turbulent stress tensors arise, i.e., $\tau_{i j}=\bar{u}_{i} \bar{u}_{j}-\overline{u_{i} u_{j}}$ corresponding to $G$ and $T_{i j}=\hat{\bar{u}}_{i} \hat{\bar{u}}_{j}-\widehat{u_{i} u_{j}}$ based on the composition filter $H \star G$ where $\star$ denotes the convolution product. Upon introducing the resolved turbulent stress tensor,

$$
\mathcal{L}_{i j}=\hat{\bar{u}}_{i} \hat{\bar{u}}_{j}-\widehat{\widehat{u_{i} u_{j}}},
$$

it is straightforward to verify the well-known Germano identity,

$$
T_{i j}-\hat{\tau}_{i j}=\mathcal{L}_{i j} .
$$

This identity is at the heart of the dynamic procedure and is used to dynamically determine model coefficients that may be contained in specific basic subgrid models, such as the Smagorinsky parameter in $m_{i j}^{S}$. The standard dynamic procedure proceeds by assuming that $\tau_{i j}$ and $T_{i j}$ can be approximated with the same model, i.e.,

$$
\tau_{i j} \rightarrow m_{i j}(\overline{\mathbf{u}}) \text { and } T_{i j} \rightarrow m_{i j}(\hat{\overline{\mathbf{u}}}) .
$$

Corresponding to this, the left-hand side in (7) is approximated by $\mathcal{M}_{i j}$ which can be written in detail as

$$
\mathcal{M}_{i j}=m_{i j}(\hat{\overline{\mathbf{u}}})-\widehat{m_{i j}(\overline{\mathbf{u}})} .
$$

When $m_{i j}$ is an appropriate model for the subgrid scale stresses, the resulting $\mathcal{M}_{i j}$ should correspond well to $\mathcal{L}_{i j}$. Since $\mathcal{L}_{i j}$ can be calculated from the resolved scales in an actual LES, a set of consistency relations is obtained, which can be used as guidance to locally and dynamically determine any model parameters in the assumed subgrid model by optimizing $\left|\mathcal{L}_{i j}-\mathcal{M}_{i j}\right|$ in an appropriate least-squares approach. ${ }^{10}$ In principle, several model coefficients that may be contained in a specific subgrid model can be determined simultaneously during a simulation. In practice, virtually always only one model coefficient is determined.

In line with the basic Smagorinsky eddy-viscosity model, the dynamic procedure starts by putting

$$
\begin{aligned}
& m_{i j}(\overline{\mathbf{u}})=2 C_{d} \bar{\Delta}^{2}|\bar{S}| \bar{S}_{i j}=2 \ell_{d}^{2}|\bar{S}| \bar{S}_{i j}, \\
& m_{i j}(\hat{\overline{\mathbf{u}}})=2 \alpha^{2} C_{d} \bar{\Delta}^{2}|\hat{\bar{S}}| \hat{\bar{S}}_{i j}=2 \alpha^{2} \ell_{d}^{2}|\hat{\bar{S}}| \hat{\bar{S}}_{i j},
\end{aligned}
$$

where we introduced the filter ratio $\alpha=\hat{\bar{\Delta}} / \bar{\Delta}$ and assumed the dynamic model coefficient $C_{d}$ to be the same on both the $G$ and the $H \star G$ filter levels. In analogy with the basic Smagorinsky model we introduced the dynamic length scale $\ell_{d}$ $=\bar{\Delta} \sqrt{C_{d}}$, which is the only parameter that is required to result from the dynamic procedure. We will restrict ourselves to homogeneous, decaying turbulence, for which it is customary to consider $C_{d}$ to depend on time alone and hence the factor $\ell_{d}^{2}=C_{d} \bar{\Delta}^{2}$ can be taken out of the test filtering in the contribution $\widehat{m_{i j}(\overline{\mathbf{u}})}$. In total, the dynamic procedure hence provides a prediction of $\ell_{d}^{2}$ and, after the usual least-squares approach $^{10}$ one obtains 


$$
\ell_{d}^{2}=C_{d} \bar{\Delta}^{2}=\frac{1}{2} \frac{\left\langle\mathcal{L}_{i j} M_{i j}\right\rangle}{\left\langle M_{i j} M_{i j}\right\rangle},
$$

where we introduced

$$
M_{i j}=\alpha^{2}\left|\hat{\bar{S}} \hat{\bar{S}}_{i j}-\right| \widehat{\bar{S}} \mid \widehat{\bar{S}}_{i j} .
$$

In (11) we denote averaging over the flow domain by $\langle\cdot\rangle$.

The dynamic procedure provides an eddy viscosity that adapts in response to the evolving turbulent flow. Rather than having to externally specify a Smagorinsky constant, based on evidently incomplete heuristical knowledge about the underlying turbulent flow, the dynamic procedure generates a "Germano-optimal" value for $\ell_{d}^{2}$ in accordance with the flow. In order to obtain the dynamic length scale $\ell_{d}$ the test filter $H$ and the filter ratio $\alpha$ need to be specified, to which we turn next.

It is quite common in LES practice that the basic LES filter $G$ is left unspecified. This leads to the principal problem that $\alpha$ is unknown. Instead of addressing this theoretical issue, we will take a more pragmatic approach and consider the filter ratio to be an external parameter. In the sequel, we will investigate the robustness of the predictions in relation to $\alpha$. For the test filter $H$, a top-hat or a Gaussian filter will be considered. The numerical integrations will be evaluated with the trapezoidal rule in order to obtain a representative implementation. Finally, we need to specify $\hat{\Delta}$ for which we adopt $\hat{\Delta}=\alpha h$ thus following several dynamic procedure implementations in literature. ${ }^{8,10,12,20}$ With these choices all required details of the specific dynamic procedure used in this study are available. Slightly different alternatives have been used as well ${ }^{21}$ but incorporating these would not change the main conclusions of our study.

We will quantify the error behavior in terms of the accuracy chart that was introduced in Ref. 16. For the Smagorinsky fluid the accuracy chart can be expressed using the resolution of the Smagorinsky length $\xi_{S}=\ell_{S} / h$ and the spatial resolution $N$ as basic parameters. The error landscape that arises from systematic variation of $\left(\xi_{S}, N\right)$ also provides the optimal refinement strategy $\hat{\xi}_{S}(N)$ which identifies the optimal value of $\ell_{S}$ that leads to the smallest total error at a particular resolution. This optimal refinement strategy takes all interacting errors due to spatial discretization and subgrid modeling into account but is available only after a large set of systematically varied large-eddy simulations has been performed.

The dynamic procedure gives rise to a "build-in" value for the dynamic coefficient, consistent with the numerical and modeling parameters adopted. By repeating simulations at different $N$, a dynamic trajectory $\xi_{d}(N)=\ell_{d}(N) / h$ is obtained on which a particular total error is induced. In order to quantify the efficiency with which the dynamic procedure reduces the total simulation error upon refining the grid, one may compare the location of the optimal $\hat{\xi}_{S}(N)$ with the dynamic trajectory $\xi_{d}(N)$. For this purpose one needs to obtain a single value for the dynamic length scale in each particular simulation. However, the dynamic length scale varies slightly with time and in the actual simulation $\ell_{d}(t, N)$ is used. For the definition of the dynamic trajectory we average the dynamic length-scale over time in a post processing step, leading to

$$
\ell_{d}(N)=\frac{1}{T} \int_{0}^{T} \ell_{d}(t, N) d t,
$$

where $T$ is the total simulation time. Since the variations in $\ell_{d}(t, N)$ are quite small, ${ }^{12,14,17,20}$ the representation of the dynamic length scale by its time average is well justified for the purpose of error assessment.

\section{DYNAMIC AND OPTIMAL TRAJECTORIES}

As mentioned in the Introduction, the aim of this paper is to compare the error level in "dynamic LES" with the optimal error level possible in a Smagorinsky fluid. In particular, we will quantify this by comparing the dynamic trajectory $\xi_{d}(N)$ with the optimal setting $\hat{\xi}_{S}(N)$. As point of reference, we will first present the optimal refinement trajectories that are obtained from an extensive simulation study of homogeneous, decaying turbulence. Then we will present the dynamic trajectories, corresponding to a top-hat test filter $H$ and the commonly adopted filter ratio $\alpha=2$. The sensitivity of $\xi_{d}(N)$ in relation to $\alpha$ and $H$ is the subject of the following section.

In order to obtain the optimal refinement strategy we follow a database approach. We consider decaying homogeneous isotropic turbulence at two different Taylor Reynolds numbers; $\operatorname{Re}_{\lambda}=50$ and 100 . The numerical method is based on explicit Runge-Kutta time stepping and adopts fourthorder accurate finite volume discretization of the convective fluxes and a second-order accurate treatment of the subgrid and viscous fluxes. ${ }^{7}$ For each of the two flow regimes considered a direct numerical simulation was performed to provide an accurate point of reference, needed to define a suitable measure for the error. The Smagorinsky model was used and a set of systematically varied large-eddy simulations of such a Smagorinsky fluid was obtained in which the Smagorinsky length scale $\ell_{S}$ and the spatial resolution $N$ were varied.

To illustrate the methodology, we will restrict ourselves in the present paper to an error assessment based on the resolved kinetic energy $E(t)=\left\langle\bar{u}_{i} \bar{u}_{i}\right\rangle / 2$. The total error in $E$ resulting from "Smagorinsky LES" with parameter $\ell_{S}$ and grid spacing $h=1 / N$ is defined as

$$
e_{t o t}\left(\xi_{S}, N, t\right)=E_{D N S}(t)-E_{L E S}\left(\xi_{S}, N, t\right) .
$$

Here, $E_{D N S}$ corresponds to the reference DNS solution. This total error is time dependent. In order to arrive at a single number to characterize the error in any specific LES we introduce a global relative error as follows: ${ }^{17}$

$$
\delta_{E}^{2}\left(\xi_{S}, N\right)=\frac{\left\|e_{t o t}\right\|^{2}}{\left\|E_{D N S}\right\|^{2}}=\frac{\int_{0}^{T} e_{t o t}^{2}\left(\xi_{S}, N, t\right) d t}{\int_{0}^{T} E_{D N S}^{2}(t) d t},
$$


The precise assessment of the error dynamics depends to some degree on the error-measure that is used. In (14) we adopted the point of view that LES predictions are desired to correspond as closely as possible with unfiltered DNS data. This implies that with this particular error-measure the error is never exactly equal to zero. In fact, it always contains a contribution arising from the spatial filtering of the solution. Other choices for the basic error-measure are of course possible. A natural alternative in the context of LES would be to compare the predictions with spatially filtered DNS data, evaluated on the corresponding LES simulation grid, i.e., using $\bar{e}_{\text {tot }}=E_{\overline{D N S}}-E_{L E S}$. This error-measure would be exactly zero for the initial condition and would remain zero in the hypothetical case in which the subgrid model would be perfect and no contributions would arise from discretization errors. In Ref. 6 the effect of changing from $e_{\text {tot }}$ to $\bar{e}_{\text {tot }}$ was considered in detail. Apart from some small changes in the error landscape, the main conclusions were found to be robust against this switch in the error-measure. Adhering to the definition in (14) has the benefit that an optimal correspondence with unfiltered data can be quantified. This would also correspond to a situation in which a comparison with experimental results is desired. In such circumstances filtered data are usually not available and hence only a comparison in the context of (14) is possible.

Although $\delta_{E}$ provides a plausible error-measure to assess some important aspects of the total error dynamics in a particular large-eddy simulation, a good prediction of the turbulent kinetic energy is not sufficient to guarantee accuracy of all other flow properties. In fact, the selection of acceptable error-measures is part of the analysis and certainly may require application-specific input in some cases. To illustrate this, one might often be interested in accurately capturing not only the decay of the kinetic energy, but also desire an acceptable distribution of this energy over the different length scales. In this case, more precise and involved errormeasures may need to be considered. As an example, the energy spectrum was found to be quite satisfactory along the "optimal trajectory" using $\delta_{E}$, except for the very low resolutions $16^{3}$ and $24^{3}$. In the latter cases a considerable deviation from the DNS spectrum was observed, displaying an excessive energy pile-up at higher resolved wavenumbers of the spectrum. By introducing an error-measure that contains a weighted integral of the energy spectrum over its wavenumber range the shape of the spectrum can be taken into account and an alternative interpration of the optimal trajectory is obtained. A judicious construction of an appropriate error-measure in a particular application should be made, and to enhance the credibility of the conclusions, the sensitivity of the predictions should be properly assessed. In this paper we will not consider this further but investigate the error dynamics according to $\delta_{E}$ instead, to illustrate in detail the main methodology.

The global relative error measure $\delta_{E}$ can be used to collect the error information in a concise way, resulting in the previously mentioned accuracy charts. In fact, $\delta_{E}$ may be calculated in each point on the $\xi_{S}-N$ plane and from a contour plot of this representation one may infer the error behavior as well as determine optimal working regions. ${ }^{16}$ In

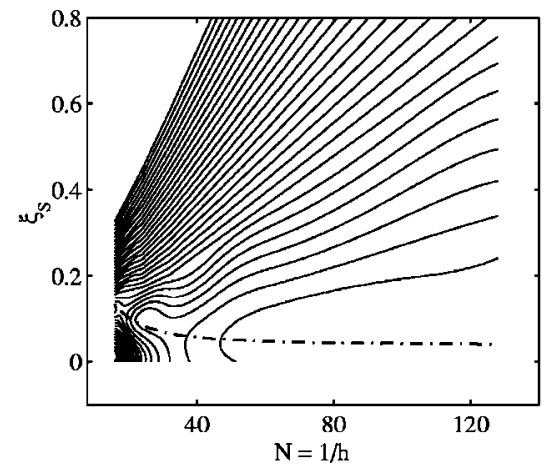

(a)

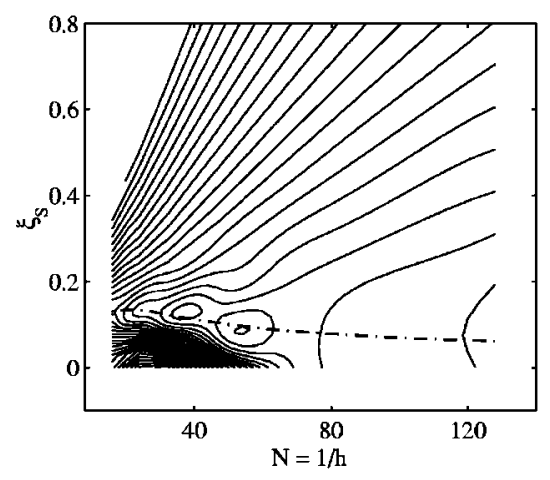

(b)

FIG. 1. Optimal refinement trajectory and accuracy chart based on $\delta_{E}[\%]$ : (a) $\operatorname{Re}_{\lambda}=50$ and (b) $\operatorname{Re}_{\lambda}=100$. Optimal refinement trajectory: (-). Contour levels start at $1 \%$ (a) $[2 \%$ (b)] at the lower right contour and increase in steps of $1 \%(2 \%)$.

Fig. 1 contour plots are presented for $\delta_{E}\left(\xi_{S}, N\right)$ from which the optimal refinement trajectories $\hat{\xi}_{S}(N)$ can be determined straightforwardly. We also observe a fairly sharp increase in the total simulation error in case the Smagorinsky parameter is sub optimal. Notice in addition that the optimal $\xi_{S}$, and hence the optimal $C_{S}$, is not constant but depends on the spatial resolution; this is due to the fact that total errors arise from an interaction between both the spatial discretization as well as from flaws in the assumed subgrid model. These errors have their own effect on the dynamics of the computational model and may be shown to counteract each other. ${ }^{16,17}$

We next turn to the error behavior associated with "dynamic-LES." Figure 2(a) represents the optimal refinement strategies for both Reynolds numbers and compares these to the dynamic trajectories. Here, the test filter $H$ is a top-hat filter with $\hat{\Delta}=2 h$, implemented using the trapezoidal integration rule. As can be appreciated in this figure, the dynamic procedure leads to a dynamic length-scale resolution $\xi_{d}$, which is somewhat too large compared to $\hat{\xi}_{S}(N)$. However, the general trend is clearly similar at both Reynolds numbers, and an effective reduction in the total error arises from grid refinement. To quantify this further, in Fig. 2(b), the errors along the optimal refinement trajectories as well as those along the dynamic trajectories are displayed. The dynamic model results are by definition suboptimal and the induced error, with respect to the kinetic energy, is seen to be roughly twice as large as the minimal error possible with a Smagorinsky fluid. Still, the dynamic procedure is 


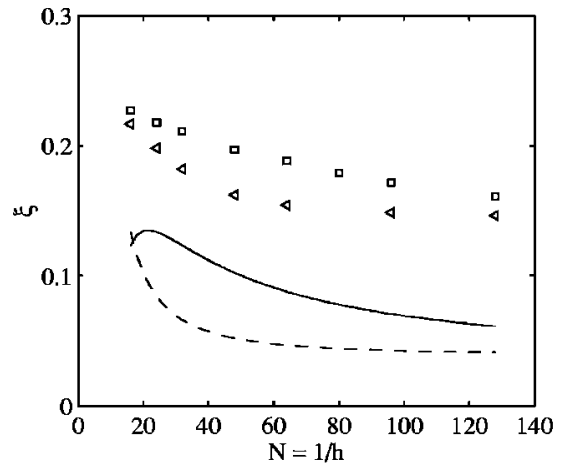

(a)

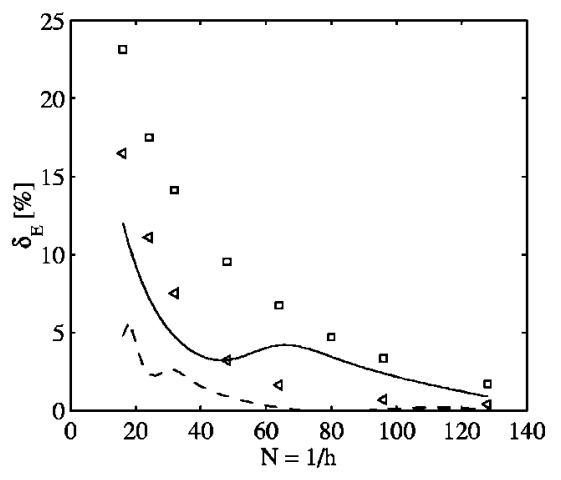

(b)

FIG. 2. (a) Optimal refinement trajectory $\hat{\xi}_{S}(N)$ vs the dynamic trajectory $\xi_{d}(N)$ obtained from the dynamic procedure. (b) Errors along the optimal and dynamic trajectories. (Both) (-), Optimal for $\operatorname{Re}_{\lambda}=100$; (--), optimal for $\operatorname{Re}_{\lambda}=50 ;(\square)$, dynamic for $\operatorname{Re}_{\lambda}=100 ;(\triangleleft)$, dynamic for $\operatorname{Re}_{\lambda}=50$.

clearly able to follow the major trends observed in the database results without requiring external ad hoc interference with the model, other than through the specification of the test-filter and the filter-ratio parameter $\alpha$. The central benefit of the dynamic model is that the response of the dynamic coefficient to an increase in the resolution follows from a build-in mechanism with which the total simulation error is seen to be reduced very effectively. For other, nondynamic models, such as the Smagorinsky model, the structurefunction model, ${ }^{22}$ the wall-adapting linear eddy model, ${ }^{23}$ or the variational multiscale method, ${ }^{24}$ the resulting simulation error is directly associated with the values that are adopted for the model parameters. In some cases, plausible values are suggested for these model parameters, based on approximate theory and assumed spectral scaling properties of turbulent flow. However, one may also systematically investigate the dependence of the total error on these parameters by varying them as ad hoc simulation parameters. This would then lead to the corresponding accuracy chart for that particular model following Ref. 16. At present, only such a systematic, extensive, and hence costly database approach allows to determine the optimal model-parameter variations as function of the spatial resolution, for a particular numerical method. It is certainly very meaningful to repeat this database approach for these interesting subgrid models. This is the subject of future research.

So far we used the (dynamic) Smagorinsky model to illustrate the error-classification framework as was introduced in Ref. 16. This may readily be extended, e.g., to other eddy-viscosity models by reinterpreting the resolution of the Smagorinsky length $\xi_{S}=\ell_{S} / h$. For this purpose we introduce the local turbulent eddy viscosity $\nu_{t}$ and the local turbulent dissipation rate $\varepsilon_{t}$. For the Smagorinsky model these are defined as $\nu_{t}=\ell_{S}^{2}|\bar{S}|$ and $\varepsilon_{t}=m_{i j}^{S} \bar{S}_{i j}=\ell_{S}^{2}|\bar{S}|^{3}$. Correspondingly,

$$
\ell_{S}=C_{s} \bar{\Delta}=\left(\frac{\nu_{t}^{3}}{\varepsilon_{t}}\right)^{1 / 4} \text {. }
$$

This relation resembles the definition of the "Kolmogorov dissipation length," albeit without statistical averaging and based on the subgrid-scale-model viscosity and dissipation. Correspondingly, we may identify $\ell_{S}$ as the Kolmogorov dissipation length for the particular subgrid model.

The reinterpretation of $\ell_{S}$ suggests to define a dissipation length $\ell_{s g s}$ for other eddy-viscosity models as well. To this end, we first introduce a "local dissipation length" $\tilde{\ell}_{s g s}$ through $\tilde{\ell}_{s g s}=\left(\nu_{t}^{3} / \varepsilon_{t}\right)^{1 / 4}$ and define $\ell_{s g s}=\left\langle\widetilde{\ell}_{s g s}^{4}\right\rangle^{1 / 4}$ where $\langle\cdot\rangle$ denotes ensemble averaging. Note that $\tilde{\ell}_{s g s}$ may vary in space and/or time while $\ell_{s g s}$ is a characteristic length scale. The error-dynamics for different eddy viscosity models may now be established in a systematic way in terms of the resolution of the corresponding dissipation length scale, i.e., $\xi_{s g s}=\ell_{s g s} / h$. For the Smagorinsky model, one readily establishes that $\tilde{\ell}_{s g s}=\ell_{s g s}=\ell_{S}$. As another example, the Kolmogorov scaling eddy-viscosity model ${ }^{25}$ corresponds to $\nu_{t}$ $=C_{K} \bar{\Delta}^{4 / 3}\left\langle\varepsilon_{t}\right\rangle^{1 / 3}$ in terms of a parameter $C_{K}$. This model implies $\ell_{s g s}=C_{K}^{3 / 4} \bar{\Delta}$, which fully corresponds to the Smagorinsky length $\ell_{S}$ in case we select $C_{S}=C_{K}^{3 / 4}$. The dynamic procedure is applied to obtain $C_{K}\left\langle\varepsilon_{t}\right\rangle^{1 / 3}$. For homogeneous isotropic turbulence, the decay of the kinetic energy was found to coincide to within line thickness for these two dynamic models, while differences in the dynamic coefficient $C_{d}$ and the equivalent value obtained for the dynamic Kolmogorov scaling model, are maximally about $5 \%$ (see also Ref. 25). Translated into the resolution of the corresponding dissipation length scales of both dynamic models, the differences in the dynamic trajectories will also be in this range. The sensitivity of the dynamic trajectories to switching among these specific eddy-viscosity models hence appears very limited.

In the following section, we will concentrate further on the dynamic procedure by evaluating the "static" and dynamic Smagorinsky model, using the proposed methodology. Main implementation choices of the dynamic procedure, such as the test-filter ratio and the test-filter shape are investigated in detail.

\section{INFLUENCE OF FILTER-RATIO AND TEST-FILTER SHAPE ON DYNAMIC TRAJECTORY}

In this section we will consider the influence of variations in the shape of the test filter and the filter ratio $\alpha$, on the quality of the dynamic LES predictions. We will first turn attention to a top-hat test filter and vary $\hat{\Delta}=\alpha h$. We will show that the accuracy of the predictions improves if $\alpha$ is increased and that the dynamic refinement trajectories show converge to an $\alpha$ independent asymptote for $\alpha \geqslant 4-6$. Subsequently, we will investigate the consequences of changing 


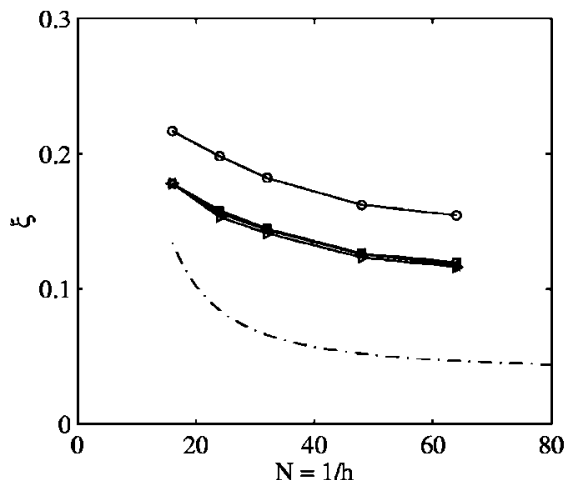

(a)
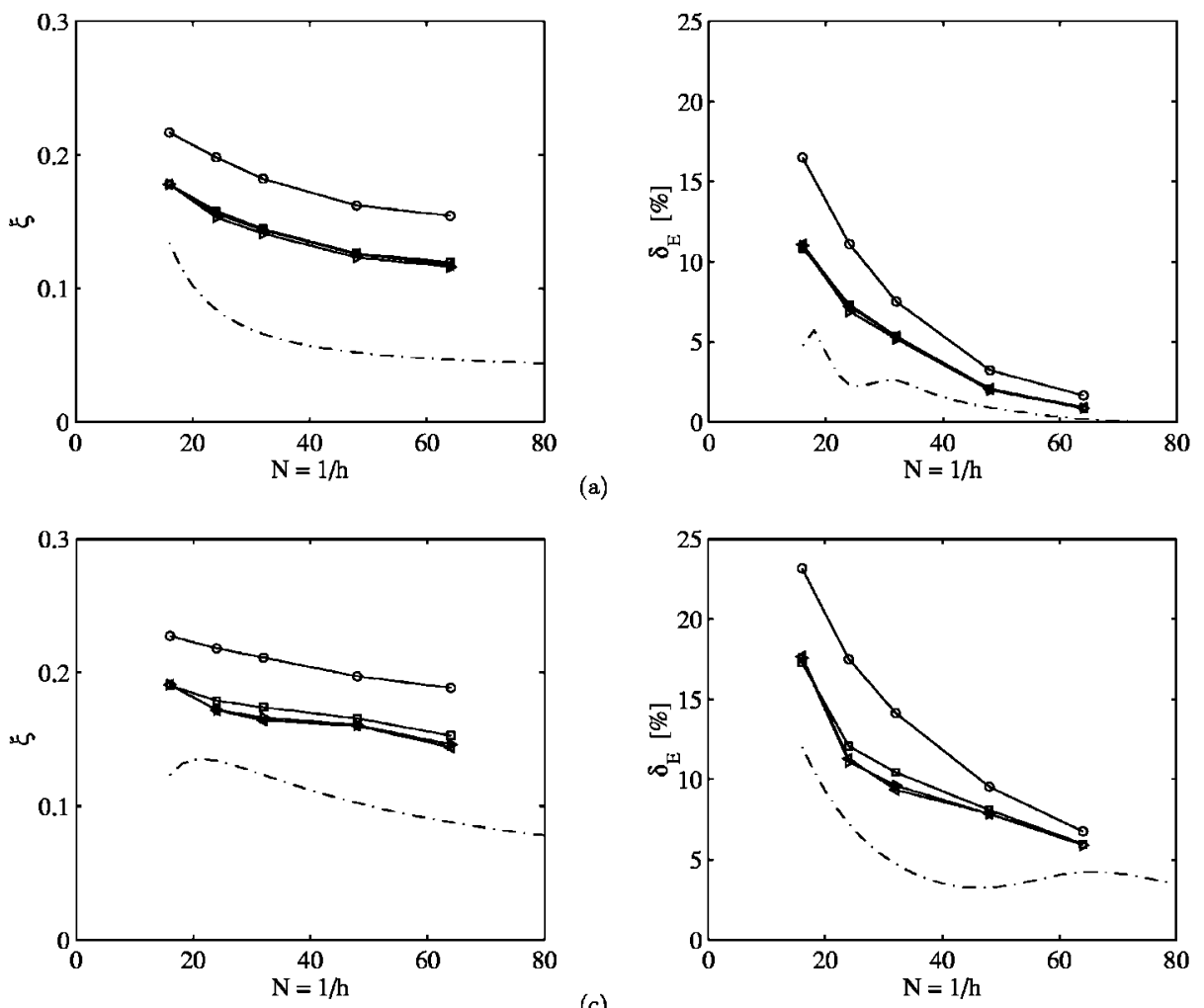

(c)

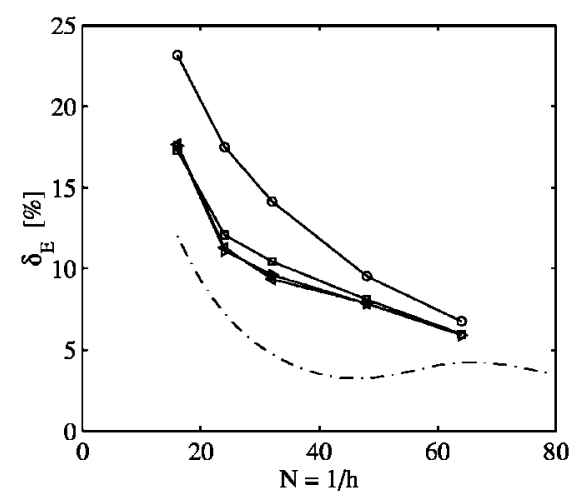

FIG. 3. Variation of dynamic results as function of $\alpha$ and $N$. (a) $\xi_{d}(N)$ for $\operatorname{Re}_{\lambda}=50$. (b) $\delta_{E}(N)$ for $\operatorname{Re}_{\lambda}=50$. (c) $\xi_{d}(N)$ for $\operatorname{Re}_{\lambda}=100$. (d) $\delta_{E}(N)$ for $\operatorname{Re}_{\lambda}=100 .(\ominus), \alpha=2 ;(\boxminus), \alpha=4 ;(\ominus)$, $\alpha=6 ; \quad(\varangle), \quad \alpha=8 ; \quad(-), \quad$ optimal trajectory. the test filter into a Gaussian filter. Here, the observed $\alpha$ convergence is less pronounced but a significant error reduction with increasing $\alpha$ may be obtained at appropriate spatial resolution.

It is common in LES to choose the width of the test filter $\hat{\Delta} \geqslant 2 h$ and therefore we restrict to $\alpha \geqslant 2$. In Fig. 3 we collected results for the sensitivity in the dynamic trajectories and the error induced on these trajectories, for both Reynolds numbers and a number of spatial resolutions $N^{3}$ with $N$ $=16, \ldots, 64$. In Figs. 3(a) and 3(c) the variations in the dynamic trajectories are collected. We observe that the dynamic length-scale resolution $\xi_{d}(N)$ is predicted to be larger than the optimal value $\hat{\xi}_{S}(N)$ for all values of $\alpha$. In Figs. 3(b) and $3(\mathrm{~d})$ the corresponding variations in the global relative error $\delta_{E}$ are compared with the optimal refinement strategy. It is appreciated that an increase of the filter ratio from $\alpha=2$ to $\alpha=4$ results in a significant decrease in error and a better approximation of $\hat{\xi}_{S}$. Moreover, for $\alpha \geqslant 4$ [in case of $\operatorname{Re}_{\lambda}$ $=50$ ], and $\alpha \geqslant 6$ [in case of $\operatorname{Re}_{\lambda}=100$ ], the dynamic trajectories virtually coincide.

Apart from varying $\alpha$, i.e., the test-filter width, it is important to investigate variations in dynamic LES results associated with a change in test-filter shape. To this end, the dynamic procedure is also performed using a Gaussian filter. The numerical evaluation of the test filter is based on the trapezoidal integration rule. In contrast to the top-hat filter, the Gaussian filter does not have a compact support which implies that the numerical integration needs to be applied to a sufficiently wide integration domain. The Gaussian kernel itself has a length scale $\hat{\Delta}=\alpha h$ and in order to evaluate this filter in a grid point $x_{j}$ we use numerical integration from $x_{j}-2 \hat{\Delta}=x_{j-2 \alpha}$ to $x_{j}+2 \hat{\Delta}=x_{j+2 \alpha}$ in the present implementation. This integration domain was found to be sufficiently wide for our purposes.

Especially at coarse resolutions and large values of $\alpha$ the integration domain for the Gaussian filter corresponds to a large portion of the flow domain and may even be larger than the integral length scale of the turbulence, which is about half the box size. ${ }^{16}$ In total $4 \alpha$ grid points in each coordinate direction are included. In contrast, the top-hat test filter with filter width $\hat{\Delta}=\alpha h$ requires only $\alpha$ grid points in each direction. Since the top-hat and Gaussian filters are both secondorder test filters, i.e., $\hat{u}=u+O\left(\hat{\Delta}^{2}\right)$, some degree of similarity in the predictions may be expected. However, although the Gaussian kernel is well localized in physical space, the contributions from grid points outside the immediate neighborhood of width $\hat{\Delta}$ can not be neglected and will be the main source for possible differences in the LES predictions. This effect may be expected to be largest at coarser resolutions and larger $\alpha$ values. We will consider the differences in more detail next.

In Figures 4(a) and 4(b) results are presented for the dynamic procedure based on a Gaussian test filter, where $\xi_{d}(N)$ and $\delta_{E}(N)$ are displayed, respectively, for different filter ratios at $\operatorname{Re}_{\lambda}=100$. Results corresponding to $\operatorname{Re}_{\lambda}=50$ are quite similar and are not included explicitly for this reason. From these figures, it is, on the one hand, clear that at the coarser resolutions and larger $\alpha$ values the trends differ considerably from those associated with the top-hat filter [cf. Figs. 3(c) and 3(d)]. On the other hand, at higher resolutions $(N=64)$ the values for $\xi_{d}$ do compare quite well with those 


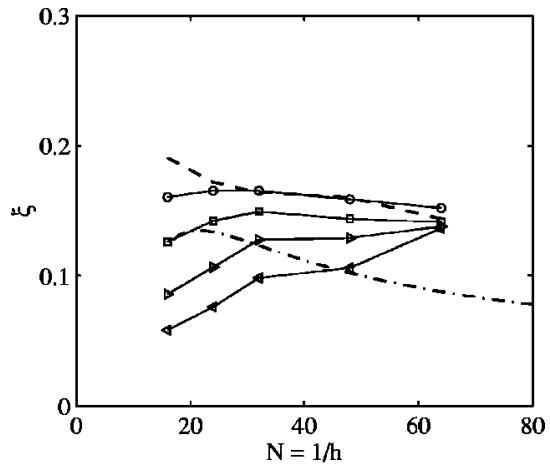

(a)

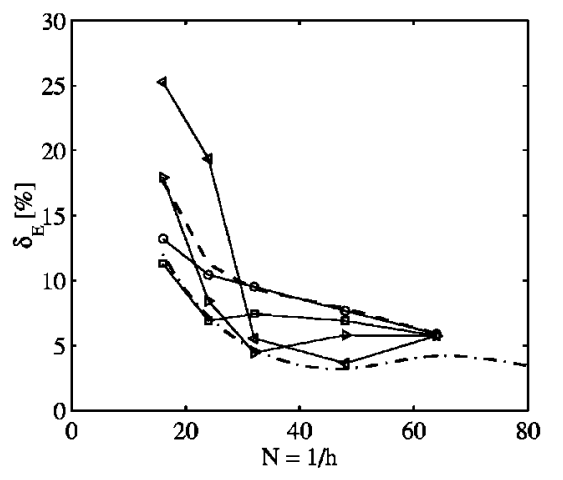

(b)

FIG. 4. Dynamic procedure with a Gaussian test filter: Variation of dynamic results as function of $\alpha$ and $N$ for $\operatorname{Re}_{\lambda}=100$. (a) $\xi_{d}(N)$. (b) $\delta_{E}(N)$. (Ө), $\alpha$ $=2 ;(\boxminus), \alpha=4 ;(\nexists), \alpha=6 ;(\varangle), \alpha=8 ;(-\cdot)$, optimal trajectory; (--;), $\alpha$-independent $(\alpha=8)$ trajectory for top-hat-filter dynamic procedure (cf. Fig. 3).

found for the top-hat filter and variation with $\alpha$ is comparably limited.

The strong variations at coarser resolutions and larger $\alpha$ values appear to be related to the wide extent of the integration domain and the associated strong numerical discretization influences mentioned above. At higher resolution the induced total simulation error on the dynamic trajectories is seen to be lower for the Gaussian filter compared to the top-hat filter but these differences are only very small. In contrast, at low resolutions, the numerical evaluation aspects of the Gaussian filter are seen to lead to a clear underestimation of the Smagorinsky length compared to the optimal value. Particularly at low resolutions, the underestimation of the Smagorinsky length is accompanied with high simulations errors. This is in line with observations for the static Smagorinsky model, ${ }^{16}$ where-at low resolutions-errors are found to increase drastically when the Smagorinsky length is underestimated (see also Fig. 1).

In Fig. 4(b) the induced error is in some cases seen to be slightly lower than the optimal value. This may be attributed to the specific representation of the results used here. Any dynamic LES is represented by its time-averaged dynamic coefficient while the actual simulations adopt the instantaneous value that results from the dynamic procedure. Although these variations are relatively small the dynamic procedure can evidently provide slightly better results than a static model that uses just the time-averaged value for the eddy-viscosity coefficient. Nevertheless, within relatively small tolerances, the optimal refinement trajectory, clearly serves as a line for minimum simulation error, as can readily be appreciated from Figs. 4(a) and 4(b).

\section{CONCLUDING REMARKS}

The use of optimal refinement trajectories ${ }^{16}$ as a point of reference for the evaluation of the dynamic procedure was demonstrated. Optimal refinement trajectories for a Smagorinsky fluid may be obtained from an extensive DNS-LES database approach. These refinement trajectories provide optimal model parameters as function of resolution, for the selected combination of numerical methods, flow conditions and subgrid-scale model.

In case the top-hat filter is adopted for the test filtering, results show that the dynamic procedure provides a dynamic trajectory, which follows the main Reynolds number and resolution trends seen in the optimal refinement trajectories relatively well. However, typically an overprediction of the optimal resolution of the Smagorinsky length scale is obtained, which leads - for a wide range of parametersettings - to errors (based on turbulent kinetic energy) about twice as high as the optimal errors.

A sensitivity analysis, studying the influence of the testto-LES-filter ratio $\alpha$ and the test-filter shape, was also considered. For a top-hat test filter, $\alpha$ independence of the dynamic trajectories was observed for $\alpha \gtrsim 4$. A significant reduction of the simulation error is observed when $\alpha$ was increased from two to four. The Gaussian test filter did not display such clear $\alpha$ convergence over the full resolution range. This may be attributed to the nonlocality of the test filter, with integration stencils that at the larger $\alpha$ values and the coarser grids may even approach the box size or the integral length scale. For high-resolution grids $(N=64)$, near $\alpha$ independence of the dynamic trajectory is observed. This sensitivity analysis suggests the use of test filters which are sufficiently localized in physical space together with the choice $\alpha \geqslant 4$.

The current results clearly show the quality of a dynamic procedure, but also reveal some sensitivity to implementation choices (e.g., test-filter construction) and discretization errors. This can introduce a certain level of uncertainty in the results. Obviously, different test filters and discretization schemes can be considered in the same framework. This may be used to explore the total simulation error corresponding to that particular implementation. Besides, alternate meaningful error measures may be considered apart from the error based on the turbulent kinetic energy. Guided by these observations one may infer the need for further development of the dynamic procedure, especially such that implementation and discretization effects might be in some way accounted for. This is subject of future research.

\section{ACKNOWLEDGMENTS}

This research was performed in the framework of FWOproject under Grant No. G.0130.02. The authors acknowledge the financial support by FWO-Vlaanderen and further acknowledge support from NCF (National Computing Foundation) to make the extensive simulations possible. 
${ }^{1}$ P. Sagaut, Large Eddy Simulations for Incompressible Flows, 2nd ed. (Springer, Berlin, 2002).

${ }^{2}$ B. J. Geurts, Elements of Direct and Large-Eddy Simulation (Edwards, Flourtown, 2003).

${ }^{3}$ B. J. Geurts and D. D. Holm, "Regularization modeling for large-eddy simulation," Phys. Fluids 15, L13 (2003).

${ }^{4}$ J. Smagorinsky, "General circulation experiments with the primitive equations," Mon. Weather Rev. 91, 99 (1963).

${ }^{5}$ D. K. Lilly, "The representation of small-scale turbulence in numerical simulation experiments," Proceedings of IBM Scientific Computing Symposium on Environmental Sciences, edited by H. H. Goldstine (IBM Data Processing Division, White Plains, New York, 1967), pp 195-210

${ }^{6}$ S. B. Pope, Turbulent Flows (Cambridge University Press, Cambridge, 2000).

${ }^{7}$ B. Vreman, B. Guerts, and H. Kuerten, "Large-eddy simulation of the turbulent mixing layer," J. Fluid Mech. 339, 357 (1997).

${ }^{8}$ M. Germano, U. Piomelli, P. Moin, and W. H. Cabot, "A dynamic subgridscale eddy viscosity model," Phys. Fluids A 3, 1760 (1991).

${ }^{9}$ M. Germano, "Turbulence: The filtering approach," J. Fluid Mech. 238, 325 (1992).

${ }^{10}$ D. K. Lilly, "A proposed modification of the Germano subgrid-scale closure method," Phys. Fluids A 4, 633 (1992).

${ }^{11} \mathrm{~K}$. Horiuti, "A new dynamic two-parameter mixed model for large-eddy simulation," Phys. Fluids 9, 3443 (1997).

${ }^{12}$ P. Moin, K. Squires, W. Cabot, and S. Lee, "A dynamic subgrid-scale model for compressible turbulence and scalar transport," Phys. Fluids A 3, 2746 (1991).

${ }^{13}$ B. Vreman, B. J. Guerts, and H. Kuerten, "Large-eddy simulation of the temporal mixing layer using the Clark model," Theor. Comput. Fluid Dyn. 8, 309 (1996).
${ }^{14}$ S. Ghosal, T. S. Lund, P. Moin, and K. A. Akselvoll, "A dynamic localization model for large-eddy simulation of turbulent flows," J. Fluid Mech. 286, 229 (1995).

${ }^{15}$ J. Jiménez and R. D. Moser, "Large-eddy simulations: where are we and what can we expect?" AIAA J. 38, 605 (2000).

${ }^{16}$ J. Meyers, B. J. Geurts, and M. Baelmans, "Database-analysis of errors in large-eddy simulation," Phys. Fluids 15, 2740 (2003).

${ }^{17}$ B. J. Geurts and J. Fröhlich, "A framework for predicting accuracy limitations in large eddy simulations," Phys. Fluids 14, L41 (2002).

${ }^{18}$ O. J. McMillan and J. H. Ferziger, "Direct testing of subgrid-scale models," AIAA J. 17, 1340 (1979).

${ }^{19}$ J. W. Deardoff, "A numerical study of three-dimensional turbulent channel flow at large Reynolds numbers," J. Fluid Mech. 41, 453 (1970).

${ }^{20}$ E. T. Spyropoulos and G. A. Blaisdell, "Evaluation of the dynamic subgrid-scale model for large eddy simulations of compressible turbulent flows," AIAA, Tech. Report No. 95-0355 1995.

${ }^{21}$ B. Vreman, B. J. Geurts, and H. Kuerten, "On the formulation of the dynamic mixed subgrid-scale model," Phys. Fluids 6, 4057 (1994).

${ }^{22} \mathrm{M}$. Lesieur and O. Métais, "New trends in large-eddy simulations of turbulence," Annu. Rev. Fluid Mech. 28, 45 (1996).

${ }^{23}$ F. Nicoud and F. Ducros, "Subgrid-scale stress modelling based on the square of the velocity gradient tensor," Flow, Turbul. Combust. 62, 183 (1999).

${ }^{24}$ T. J. R. Hughes, L. Mazzei, and K. E. Jansen, "Large eddy simulation and the variational multiscale method," Computing and Visualization in Science 3, 47 (2000).

${ }^{25}$ G. Dantinne, H. Jeanmart, G. S. Winckelmans, V. Legat, and D. Carati "Hyperviscosity and vorticity-based models for subgrid scale modeling," Appl. Sci. Res. 59, 409 (1998). 